\title{
Introduction to Distributed Ledger Technology, Blockchain Minitrack
}

\author{
Isabell Welpe \\ Technical University of \\ Munich \\ welpe@tum.de
}

\author{
Liudmila Zavolokina \\ University of Zurich \\ zavolokina@ifi.uzh.ch
}

\author{
Helmut Krcmar \\ Technical University of \\ Munich \\ krcmar@in.tum.de
}

\author{
Marcus Müller \\ Technical University of \\ Munich and MIT \\ marcusmu@mit.edu
}

Among other promising technologies, Distributed Ledger Technologies (DLTs), often referred to as Blockchain, promise to be one of the most disruptive technologies since the invention of the internet's TCP/IP protocol, yet this technology so far lacks scholarly attention and coverage in peer-reviewed literature. The combination of some DLT characteristics, e.g. accountability, pseudonymity or distributed network topology, as well as the first cryptocurrency Bitcoin, drew enormous attention given the ubiquitous amount of possibilities for which this technology can be used since its invention in 2008 by Satoshi Nakamoto. Whereas TCP/IP lowered the cost of transferring data between two parties dramatically, DLTs have the potential to reduce the cost of transactions (i.e. transfer of assets and value) dramatically. DLTs can enable the secure transfer of any asset worldwide with nearly instant accountability by specifically cutting out intermediary trust holders due to the capabilities of the network's proof-of-X trust-building-processes, e.g. the Bitcoin proof-of-work mining concept. More recent developments that build on top of DLTs, like smart contracts and Decentralized Autonomous Organizations (DAOs) take the possibilities of programmable secure transactions even further.

In this context, companies, governments, and whole industries are facing dramatic organizational change and transformation processes with regard to their value chains while adopting DLTs. This minitrack welcomes fundamental research regarding methods and techniques, issues, and key challenges, as well as organizational approaches for understanding the potential of DLTs for business models, value chains, emerging competitive landscapes and new start-ups employing this technology. Research may cover any industry or organizational form and may focus on the technology layer or strategic organizational challenges, opening this track both to the IT and economic/management science community to reflect their increasing dependency on each other. Topics of interest include, but are not limited to:
- Current state of the art of blockchain technologies and smart contracts

- Applications of blockchain technology and their potential in different areas (finance, insurance, healthcare and pharmaceuticals, energy sector, manufacturing, transportation, automotive industry, provenance, government sector etc.)

- Blockchain and Internet of Things (machine-to-machine interaction, automated devices, blockchain for metered appliances, etc.)

- Impact on business models (change of existing business models, emergence of new business models, disruptive business models, etc.)

- Organizational transformation through blockchain technology (distributed autonomous organizations, etc.)

- Impact on the value chain (disintermediation, change of actors, advantages and disadvantages, etc.), the internet and the digital economy.

- Regulatory aspects of blockchain technology and implications for risk management

- Technical issues of blockchain technology (emergent protocols, consensus mechanisms, scalability, reliability, security, challenges of implementation, etc.)

- Philosophical issues on use of blockchain technology (governance of blockchain society, decentralization of society, building of community and collaboration)

Papers included in the minitrack cover the following topics:

1. Blockchain Technologies from the Consumers' Perspective: What Is There and Why Should Who Care?

2. A Model-driven Approach for the Description of Blockchain Business Networks 
3. Cross-Organizational Workflow Management Using Blockchain Technology - Towards Applicability, Auditability, and Automation

4. Disrupting Industries with Blockchain: The Industry, Venture Capital Funding, and Regional Distribution of Blockchain Ventures

5. Dynamics of Blockchain Implementation A Case Study from the Energy Sector
6. A structured framework to assess the business application landscape of blockchain technologies.

7. Unveiling the Key Challenges to Achieve the Breakthrough of Blockchain: Insights from the Payments Industry

8. Breaking Bad: De-Anonymising Entity Types on the Bitcoin Blockchain Using Supervised Machine Learning 